\title{
Development of the Manufacturing Geometry of Conical and Cylindrical Worms by Analysing of Their Axoids
}

\section{Zsuzsanna Balajti}

Faculty of Mechanical Engineering and Informatics, University of Miskolc. Egyetemváros A/4 225, H-3515 Miskolc. Hungary. E-mail: balajtizs@uni-miskolc.hu, balajtizsuzsanna@gmail.com

In this paper an examination has been outlined a possibility of further generalization respecting the Dudás's kinematic-mathematical model, which is suitable for the production geometric development of elements of helicoid drive pairs. The positioning of the rolling surfaces of the cylindrical and conical surfaces, as axoids of worms, in the extended model to the projective space under the right conditions, based on the surfaces invariant to projective transformations can be a right extension of the model, as the projective space model includes the Euclidean space model in full. The machining of the conical worm surfaces modelled in this method involves the clarification of the kinematic-geometric relations of production geometry in the Euclidean space model, which shows the possibility of further development of production geometry.

The goal of generalization of the mathematical description is the systematization by creating central collinear projective connection between axoids (rolling cones and rolling cylinders). Managing the production geometry of the worms in a system is also a further improvement in manufacturing precision.

Keywords: Conical worm, kinematical-mathematical model, axoid, rolling surface, central collinear connection

\section{Introduction}

The developments of construction, quality assurance and manufacturing geometry in the University of Miskolc has been produced a significant field of worm drivings research for more than half a century. One of the outstanding topics of the Worm Scientific Team lead by Professor Dudás is the examination of the meshing of cylindrical and conical worm gear drives. In order to examine the geometry of the cylindrical and conical worms and their tools, the known Dudás's integrated general kinematic-mathematical model can be seen on Figure 1.

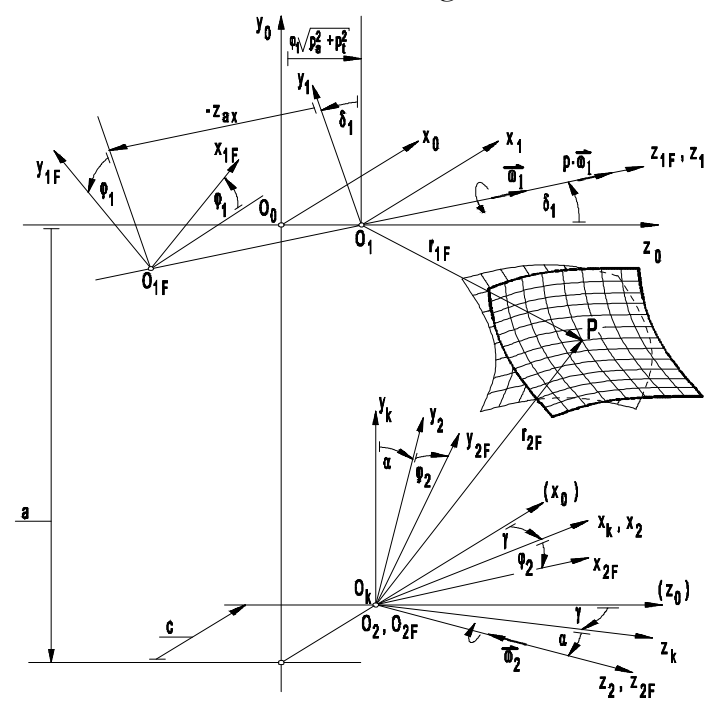

Fig. 1 General kinematic-mathematical model for the methods of the manufacturing technology [4]
The coordinate systems and applied geometrical parameters for the production geometrical analysis of the cylindrical and conical worms with the axis coinciding $z_{1 F}$, furthermore the worm-gears and the tools of worms with the axis coinciding $\mathrm{z}_{2 \mathrm{~F}}$ have been shown in Figure 1 abowe, where:

a, c...Distances of axes [mm],

a...Forming, tilting angle of tool to the helical surface in a characteristic section, e.g. grinding of an evolvent worm surface with a flat operating surface grinding wheel $\left[^{\circ}\right]$,

$\gamma$. .Lead angle on the worm's reference surface $\left[{ }^{\circ}\right]$, $\mathrm{K}_{0}\left(\mathrm{x}_{0}, \mathrm{y}_{0}, \mathrm{z}_{0}\right) \ldots$... Stacionary coordinate system [-],

$\mathrm{K}_{1 \mathrm{~F}}\left(\mathrm{x}_{1 \mathrm{~F}}, \mathrm{y}_{1 \mathrm{~F}}, \mathrm{z}_{1 \mathrm{~F}}\right) \ldots$ Rotating coordinate system affixed helicoid surface [-],

$\mathrm{K}_{1}\left(\mathrm{x}_{1}, \mathrm{y}_{1}, \mathrm{z}_{1}\right) \ldots$ Coordinate system connected to linear moving table [-],

$\mathrm{K}_{2 \mathrm{~F}}\left(\mathrm{x}_{2 \mathrm{~F}}, \mathrm{y}_{2 \mathrm{~F}}, \mathrm{z}_{2 \mathrm{~F}}\right) \ldots$ Rotating coordinate system fixed to tool [-],

$\mathrm{K}_{2}\left(\mathrm{x}_{2}, \mathrm{y}_{2}, \mathrm{z}_{2}\right) \ldots$ Stationary coordinate system fixed to tool [-],

$\mathrm{K}_{20}\left(\mathrm{x}_{20}, \mathrm{y}_{20}, \mathrm{z}_{20}\right) \ldots$ Coordinate system of the generating curve of a tool of surface of revolution [-],

$\mathrm{K}_{\mathrm{k}}\left(\mathrm{x}_{\mathrm{k}}, \mathrm{y}_{\mathrm{k}}, \mathrm{z}_{\mathrm{k}}\right) \ldots$ Auxiliary coordinate system [-],

$\mathrm{O}_{0}, \mathrm{O}_{1}, \mathrm{O}_{2}, \mathrm{O}_{1 \mathrm{~F}}, \mathrm{O}_{2 \mathrm{~F}}, \mathrm{O}_{\mathrm{k}} \ldots$.. Origins of coordinate systems related to their subscripts [-],

p... Screw parameter of the helix on worm [-],

pa... Axial screw parameter [-],

$\mathrm{p}_{\mathrm{r}} .$. Radial screw parameter [-]

$\varphi_{1} \ldots$ Angle rotation of the helical surface $\left[^{\circ}\right]$,

$\varphi_{2} . .$. Angle rotation of the tool surface $\left[{ }^{\circ}\right]$, 
$\omega_{1} \ldots$ Angular velocity of the worm $\left[\mathrm{s}^{-1}\right]$, $\omega_{2}$... Angular velocity of the tool $\left[\mathrm{s}^{-1}\right]$.

The mathematical analysis required for manufacturing geometry of elements of conical or cylindrical worm gear drives can be done in this model. During the manufacturing process, from the kinematic substitution aspect, all continuous machining can be seen as mapping of relative motions or motion informations, so the theorem of motion mapping, which is the production method of the related surfaces of special kinematic pairs, can be considered an organic part of manufacturing geometry. The analaysis of the relative motions of the drive pair's elements can be done by rigid space systems of elements, which leads to the examination of the axoids. Due to the handling of rolling cones and rolling cylinders of worms in one model, the use of projective geometry has been introduced as a generalization of the Euclidean geometry, which includes the Euclidean geometric system. In the case of modeling manufacturing geometry, in several specific cases, the Euclidean spatial model should be complemented by infinite distant space elements. $[1,4,8,14]$ The geometric analysis is performed through the invariants of the transformation groups, such as the fiting, or distance and angle. For the analysis of technical constructions, similarly to other researchers, a synthetic form of geometry has been selected to stimulate thinking, with the support by analytic geometry, continuing Petrich's professional work, who has been founded the Department of Descriptive Geometry in University of Miskolc [12].

\section{Development task}

During machining of the conical worm surface on a traditional machine with apex adjustment, the straight-line creator of the cone has been setted into parallel situation to the axis of the grinding wheel as shown in Figure 2.

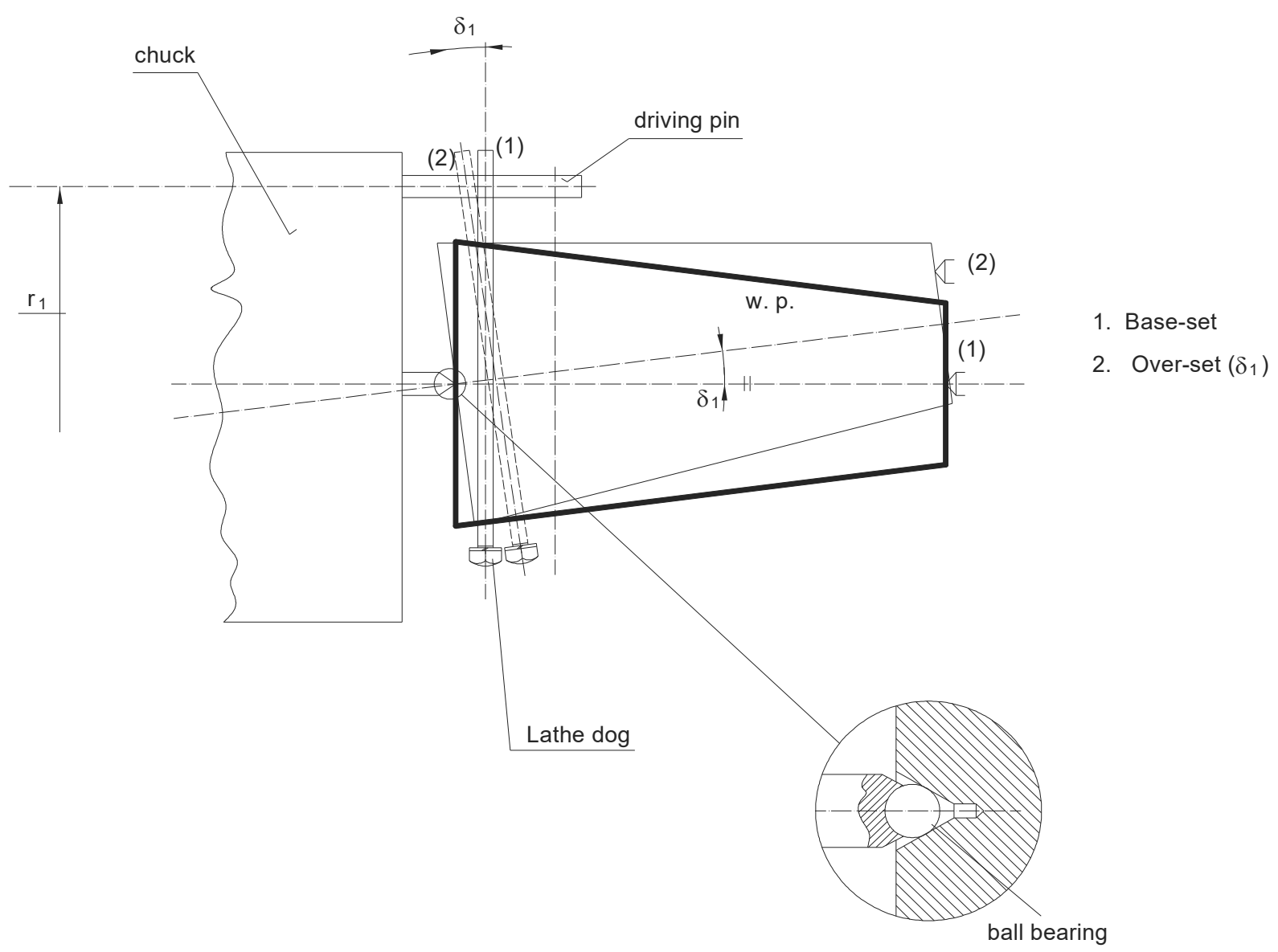

Fig. 2Shifting of the apex at the conical worm manufacturing [10]

By producef adjusting on the tailstock, the thread of the conical worm has been machined on a plane perpendicular to main spindle's axis on an elliptical orbit, due to an setted angle between the rotating main spindle and the lathe dog. In case of conical worm's machining the thread is influenced by the peripheral speed. The peripheral velocity of the chuck denoted $\mathbf{v}_{\mathrm{t}}$ can be written in the next form

$$
\mathbf{v}_{t}=r \cdot \omega
$$

The constant velocity of drive pin contact point denoted $\mathbf{v}_{\mathrm{m}}$ can be written in the next form based on Figure 3. 


$$
\mathbf{v}_{m}=r_{m} \cdot \boldsymbol{\omega}_{m}
$$

The angular velocity of workpiece denoted $\omega_{\text {mwp }}$ can be specified as

$$
\boldsymbol{\omega}_{m v p}=\frac{\mathbf{v}_{m}}{r_{2}}=\frac{r_{2} \cdot \boldsymbol{\omega}_{m}}{r_{2}} \quad \text { (variable quantity) }
$$

Variable distance from the axis changed the peripheral velocity and caused a $H$ pitch error among other problems

$$
H=p \cdot \boldsymbol{\omega}_{m w p}
$$

Depending on the angular position of the workpiece, sometimes the pitch is smaler, sometimes greater, that is, it fluctuates around a nominal value based on $p \cdot \boldsymbol{\omega}_{1}$ value.

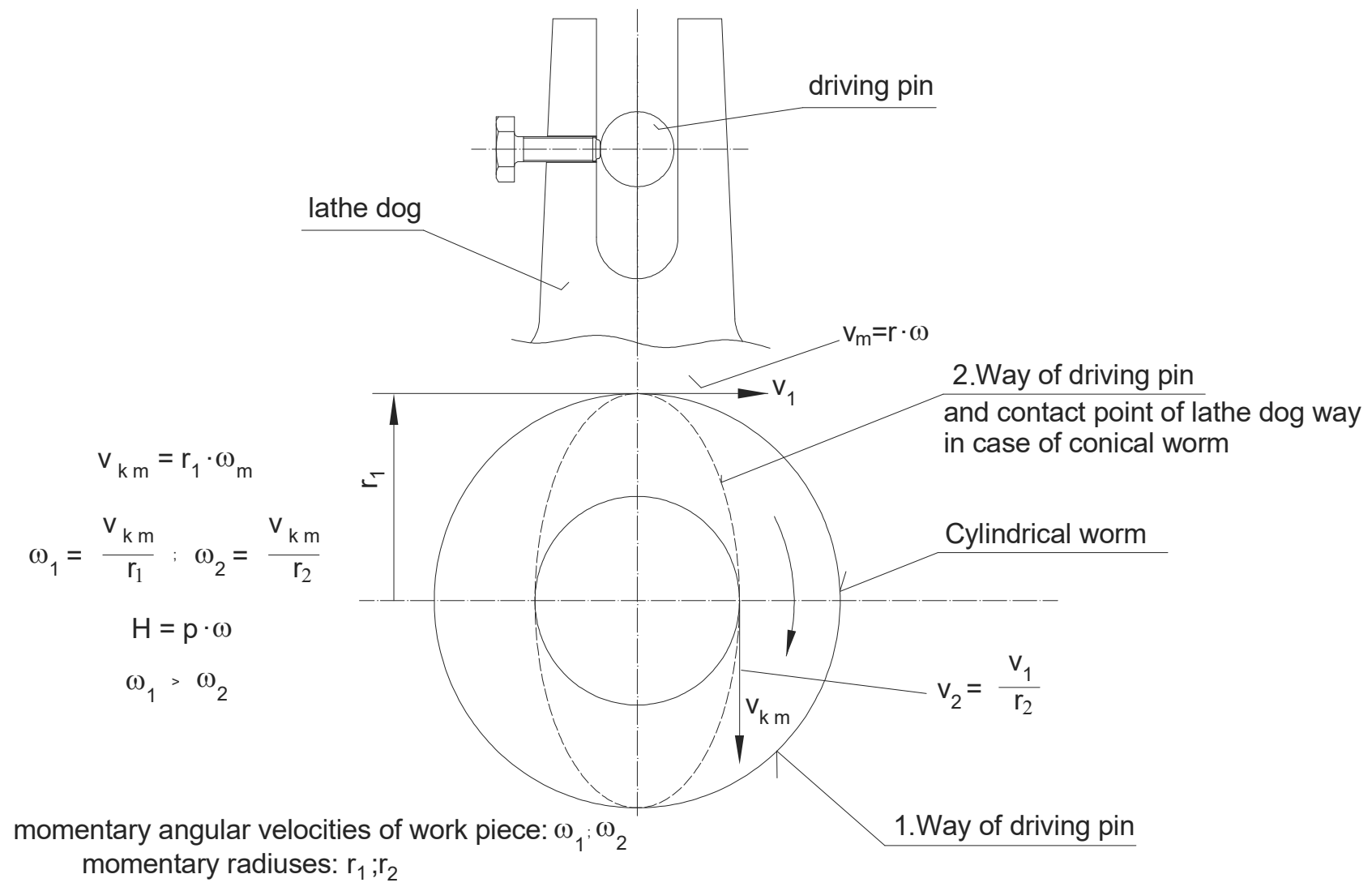

Fig. 3 Mathematical layout of the thread in case of conical worm manufacturing [10]

\section{About the axoids}

During the development of manufacturing geometry of helicoidal surfaces and their tools the necessity to investigate kinematic relationships and to treat rolling surfaces in one model is well established. [2, 4, $5,6,7,9,10,13]$

In case of the moving of the rigid plane system in its own plane, the relationship between it's two different positions can always be given by rotation around a point, or by a parallel offset to a straight line, in the case of the infinite instantaneous rotation point.

In case of the moving of the rigid three dimensional system in its own space, the relationship between it's two different positions can always be given by a helical movement along a straight line. The spatial rigid system has been descriptived by the Descartes' right-handed mutually-orthogonal normalized axis system, which is defined by it's own three non-collinear points, such as the origo $O$, as well as the unit points $E_{x}$ and $E_{y}$ on the $x$ and $y$ axes. The spatial movement of triangle $O E_{x} E_{y}$ between it's position $O_{1} E_{x 1} E_{y 1}$ and
$O_{1}(\varphi) E_{x 1}(\varphi) E_{y 1}(\varphi)$, can be replaced by one helical movement along the helical axis $c_{t}$. In the first step the direction of the helical axis $f_{t}$ can be determined as shown in Figure 4. In case of parallel offsetting with itself on the two triangles $O_{1} E_{x 1} E_{y 1}$ and $O_{1}(\varphi) E_{x 1}(\varphi) E_{y 1}(\varphi)$ onto a point in the space in so way, that one of the matched pointpairs, such as the $O_{1}$ and $O_{1}(\varphi)$ overlape each other, the triangles $O_{1} E_{x 1} E_{y 1}$ and $\underline{\underline{O}}_{1}(\varphi) \underline{\underline{E}}_{x 1}(\varphi) \underline{\underline{E}}_{1}(\varphi)$ can be placed in an overlap position with one rotation. The axis $f_{t}$ of rotation passes through on point $O_{1}=\underline{\underline{O}}_{1}(\varphi)$. The rotation-axis $f_{t}$ is created by the intersection of two planes, of which the first plane is the bisector perpendicular plane of the section $E_{x 1} \underline{\underline{E}}_{x 1}(\varphi)$, and the second plane is the bisector perpendicular plane of the section $E_{y 1} \underline{\underline{E}}_{y 1}(\varphi)$. In the second step, it is necessary to determine the exact position of the helical movement's axis $c_{t}$. The triangles $O_{1} E_{x 1} E_{y 1}$ and $O_{1}(\varphi) E_{x 1}(\varphi) E_{y 1}(\varphi)$ are projected from their original positions parallel to the direction of rotation axis $f_{t}$, onto the plane $\underline{K}_{s}$ perpendicular to the rotation axis $f_{t}$, as shown in Figure 4. 


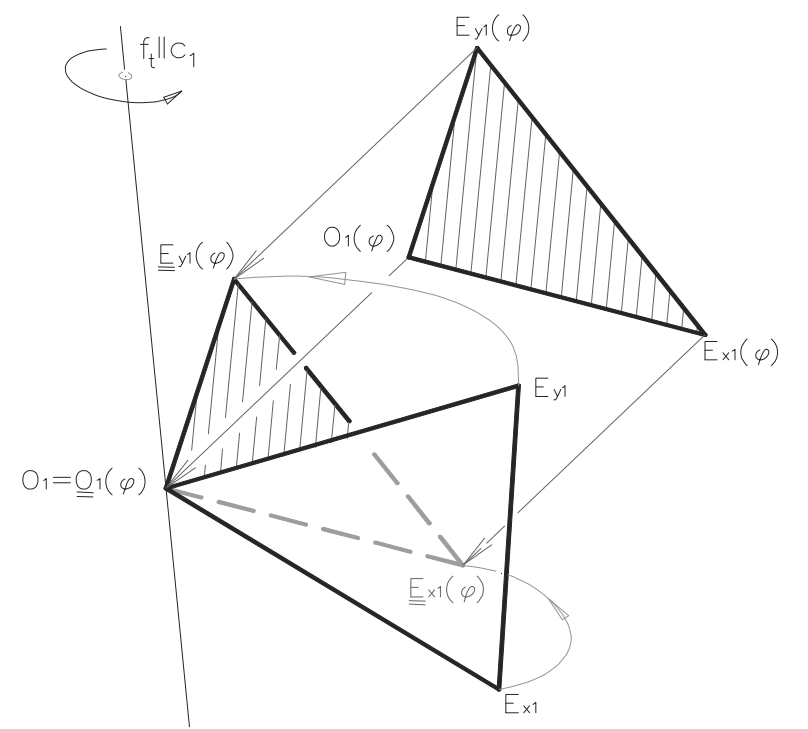

Fig. 4 Determination of the direction $f_{t}$ of the torsion axis $c_{1}$

Since the planes of the congruent triangles $O_{1} E_{x 1} E_{y 1}$ and $O_{1}(\varphi) E_{x 1}(\varphi) E_{y 1}(\varphi)$ creates an equal angle with the plane $\underline{K}_{s}$, the projections of triangles will be congruent also. In the plane $\underline{K}_{\text {s }}$ the congruent projections of triangles can be rotated into overlap with an angle $\varphi$, around an point $O$. The axis of the helical movement is a straight line $c_{t}$ parallel to the the rotation axis $f_{t}$ and passing through point $O$.

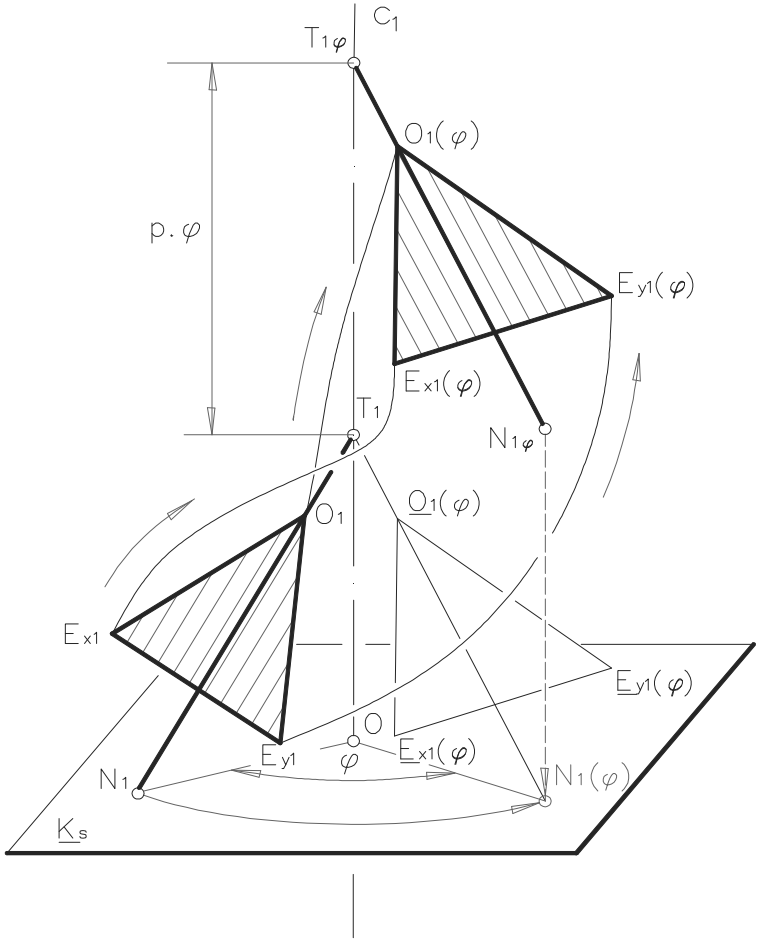

Fig. 5 The instantaneous torsion axis of motion between two spatial position

In Figure 5 the planes of the triangles $O_{1} E_{x 1} E_{y 1}$ and $O_{1}(\varphi) E_{x 1}(\varphi) E_{y 1}(\varphi)$ intersect the axis $c_{t}$ at points $T_{1}$ and $T_{2}$. The measure of displacement between points $T_{1}$ and $T_{2}$ is $p \cdot \varphi$. The triangle $O_{1}(\varphi) E_{x 1}(\varphi) E_{y 1}(\varphi)$ can be offset parallel to direction of rotation axis $f_{t}$ and the size of the section $\overline{\mathrm{T}_{1} T_{2}}$ into the intermediate position $\underline{O}_{1}(\varphi) \underline{E}_{x 1}(\varphi) \underline{E}_{y 1}(\varphi)$, which can be rotated into position $O_{1} E_{x 1} E_{y 1}$ around axis $c_{t}$, by angle $\varphi$. The resulting movement follows the helical lines described by the points $O_{1}, E_{x 1}$ and $E_{y 1}$, the common axis of the helical lines is the $c_{t}$, and the common parameter is the $p$. (If we interpret rotation angle $\varphi+2 n \pi$ instead of rotation with angle $\varphi$, then with a constant amount of offset, we get a solution with different pitch helices.)

A machine tool stand and it's any moving unit, a workpiece and tool fixed in the tool machine one by one may be considered belonging to a rigid space system.

The most general motion of the moving rigid space system $\Sigma_{1}$ correlated to the fixed rigid space system $\Sigma_{0}$ is an instantaneous screw motion. The instantaneous screw motion is determined by the instantaneous position of the instantaneous axis $c_{10}$, furthermore the instantaneous angular velocity $\omega_{10}$ of the $\Sigma_{1}$ space system correlated to $\sum_{0}$ space system, and it's instantaneous screw parameter $p_{10} \neq 0$. If the position of the screw axes $c_{10}$ in the space system $\Sigma_{0}$, the angular velocity $\omega_{10}$ and the screw parameter $p_{10}$ are constant, the screw motion is named elementary. [3]

If a space system $\Sigma_{1}$ and another spaces system $\Sigma_{2}$ are in the relative motion each other, the instantaneous screw axes of this relative motion create a line surface $\alpha_{1}$ in space system $\Sigma_{1}$ and an another line surface $\alpha_{2}$ in space system $\Sigma_{2}$. An axoid is the family of instantaneous axes of the relative motion of $\Sigma_{1}$ and $\Sigma_{2}$ three dimensional systems, that is generated in three dimensional systems $\Sigma_{1}$ or $\sum_{2}$. [8] The axoids have always appeared in pairs, they are connected (conjugated). [13]

In the space system $\Sigma_{0}$ fixed to the drive mechanism casing the screw motion space system $\Sigma_{1}$ with axis $c_{10}$, angular velocity $\omega_{10}$, parameter $p_{10}$ and also screw motion space system $\Sigma_{2}$ with axis $c_{20}$, angular velocity $\omega_{20}$, parameter $p_{20}$ are in relative motion, that is generally between in them a continuous motion of screw motions. The instantaneous screw axes create the axoid $\alpha_{1}$ in $\Sigma_{1}$ and axoid $\alpha_{2}$ in $\Sigma_{2}$, which are slipping and rolling on a distorted screw surface on each other in the most comprehensive case. These are touched together in a $\Sigma_{0}$ space system in an instantaneous relative screw axis $c_{12}=c_{21}$. In special cases the element of the axoid pairs may be a distorted screw surface, closed or opened and sharp or flat screw surfaces, a developable helicoid, a rotary distorted hyperboloid, a cylinder generated by rotation, a cone generated by rotation, ect. .

\section{About the projective spaces model}

For the analytical description of the Euclidean space, it's points are assigned a triplet of real numbers $(x, y, z)$ in the Descarte coordinate system. The homogeneous coordinates of points of the Euclidean space 
model are obtained with the following identities

$$
x=\frac{x_{1}}{x_{4}}, y=\frac{x_{2}}{x_{4}}, z=\frac{x_{3}}{x_{4}}, x_{4} \neq 0,
$$

where the $\operatorname{rang}\left(x_{1}, x_{2}, x_{3}, x_{4}\right)=1$.

Two points are identical, if their corresponding coordinates are product of scalar to each other. In case of $x_{4}=0$, the coordinates $\left(x_{1}, x_{2}, x_{3}, 0\right)$ represent the point in the infinite distance in direction $\left(x_{1}, x_{2}, x_{3}\right)$.

The homogeneous coordinates of the points fitting to the second-order surface of the projective space fulfil the next form

$$
\boldsymbol{\alpha}_{I K} \cdot x_{I} \cdot x_{K}=0 \quad(I, K=1,2,3,4)
$$

By successive application of projective transformations, it is always possible that the matrix of the second order surfaces can be made into symmetric matrix, which only has the elements in the diagonal that are different from 0 , which can be +1 and -1 . The range and signature of the matrix of the second-order surface are invariant with projective transformation. The second-order surfaces are classified according to the range and signature of their matrix representation.

Classes of second-order surfaces include surfaces with same range, denoted with $\mathrm{R}$, and with same signature, denoted with $\mathrm{S}$.

In case of $\mathrm{R}=3$ and $\mathrm{S}=1$, the second-order surface can be written by homogeneous coordinates in the next form

$$
x_{12}^{2}+x_{22}^{2}-x_{32}^{2}=0
$$

and the same surface can be written by Descartes coordinates in the Euclidean space

$$
x^{2}+y^{2}-z^{2}=0
$$

The class of the real cone surface generated by rotation also includes the rotated surface, which means that in the projective spatial model has the same qualities. Since their qualities are invariant with projective transformation, therefore projective transformation can be created between them.

The matrix form of all second order surface

$$
\mathbf{X}^{T} \cdot \mathbf{A} \cdot \mathbf{X}=0
$$

where the $\mathbf{X}^{\mathrm{T}}$ is transposed of matrix $\mathbf{X}$.

Developments in the projective space model can be linked to the Dudás' ProMat model [5].

\section{The central collineation relation between the rolling surfaces of cylindrical and co- nical worms}

Based on the Dudás mathematical-kinematical model, the Euclidean space model has been complemented by the infinite distant plane, so the rolling cone and rolling cylinder of the worms have been located in the projective space model in a central collineation relationship. In the projective space model the cylinder is a cone with an infinity vertex. To create a central collineation relationship between the two surfaces, the cone generated by rotation and the cylinder generated by rotation have been placed on the common axis $21 \mathrm{~F}$ with the following correspondence: the finite vertex $\mathrm{M}_{\mathrm{k}}$ of the cone on the axis $z_{1 \mathrm{~F}}$ and the infinite distance vertex $\mathrm{M}_{\mathrm{h}}$ of the cylinder on the axis $z_{1} \mathrm{~F}$, correspond to each other, the plane of the common circle of the cone and cylinder is the base plane As of the central collinearity, and the $\mathrm{C}$ center of the central collinearity may be any point of the axis $q_{1 F}$ without the axis point on the base plane and without the vertex of the cone and cylinder. The straight line lieing on the $\mathrm{P}_{\mathrm{h}}$ point of the cylinder and on the $\mathrm{P}_{\mathrm{k}}$ point of the cone has been passed through the center $C$. These points have been assigned to each other in the central collinear connection. The disappearing planes have been posintioned, that one of the vanishing planes has been as far as away from the center $C$ as the other vanishing plane from the base plane $\underline{\text { Ass. }}$

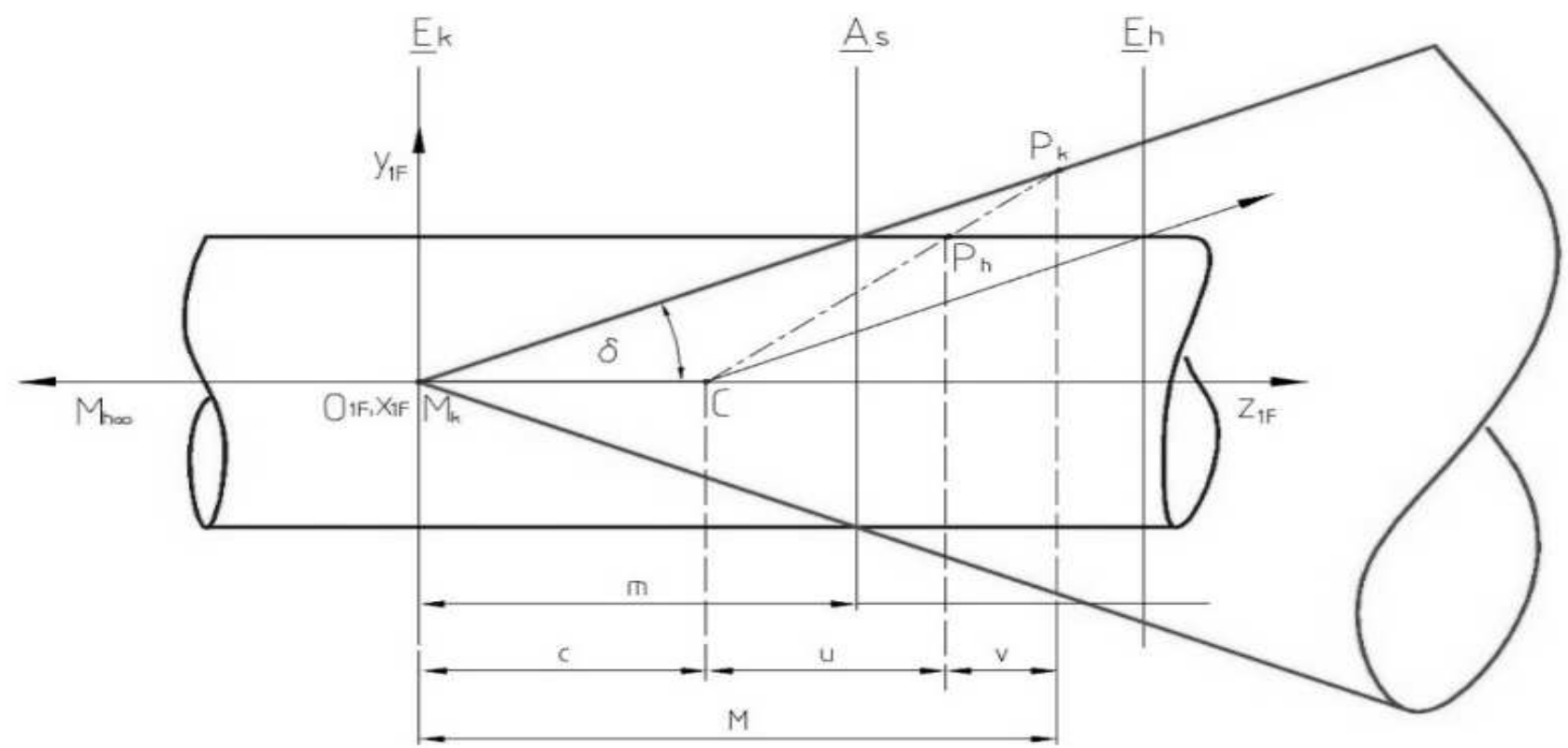

Fig. 6 Central collineation relation betwee the rolling cone and rolling cylinder of worms [11] 
The vanishing plane $\underline{E}_{k}$ of the cone fits the cone vertex $\mathrm{M}_{\mathrm{k}}$, as it can be seen on Figure 6. The coordinates of the cylinder's points (marked by $x$ ) satisfy the next equation

$$
\boldsymbol{h}_{I K} \cdot x_{I} \cdot x_{K}=0 \quad(I, K=1,2,3,4)
$$

that can be written in the following matrix format

$$
\mathbf{X}^{\mathbf{T}} \cdot \mathbf{H} \cdot \mathbf{X}=0
$$

The coordinates of the cone's points (marked by $x$ ) satisfy the next equation

$$
\boldsymbol{k}_{I K} \cdot x_{I} \cdot x_{K}=0 \quad(I, K=1,2,3,4)
$$

that can be written in the following matrix format

$$
\mathbf{X}^{\mathbf{T}} \cdot \mathbf{K} \cdot \mathbf{X}=\mathbf{0}
$$

In case of transformation from rolling cone to rolling cylinder the required identical has been fulfilled, as it can be seen in the following equation

$$
X^{T} \cdot K \cdot X=\left(X^{T} \cdot C^{T}\right) \cdot H \cdot(C \cdot X)=X^{T} \cdot\left\{C^{T} \cdot H \cdot C\right\} \cdot X
$$

where the $\mathbf{C}$ matrix represents the central collinear relationship.

\section{Creating of helicoid surfaces}

The rolling surfaces of the conical and cylindrical worms have been located in the projective space model based on their central collinearity relationship. Than the worm's teethsurfaces produced by a rotary motion and a tangential and a radial displacement in the Euclidean space model, as the Euclidean geometry is the theorem of the transformation group's invariants.

The creation of the helicoid surfaces, and it's matrix representation can be done into relation with analytical modelling of Euclidean space. An important goal is to provide usability in a single model. In the following the connection is worth examining between the locating in the projective space model of the worms' rolling surfaces and the manufacturing of the teeth surfaces in the Euclidean space model along it's matrix representations as well. The combined motion can be described by a $4 \times 4$ matrix. Placing behind the $3 \times 3$ matrix of the rotating motion the column matrix of the translation, so the $3 \times 4$ matrix has to be complemented in the bottom line with the row $[0,0,0,1]$ to a $4 \times 4$ matrix.

Handling of the coordinates of the points is also important to consider the theoretical clarification. The rolling surface of the worms can be written in the projective space using a $4 \times 4$ matrix, to which can be used the homogeneous coordinates of points. The homogeneous coordinates $\left(\lambda \cdot x_{1}, \lambda \cdot x_{2}, \lambda \cdot x_{3}, \lambda \cdot x_{4}\right),(\lambda \neq 0)$ of points can be formed by $(x, y, z)$ Descartes coordinates of points according to formula (1).

The description of the combined motions can be done with a part homogeneous coordinates $(x, y, z, 1)$ formed from the $(x, y, z)$ Destartes coordinates of points in the Euclidean space model, using only $x_{4}=1$ from the endless possibilities.

One of the importan direction of the mathematical development is to set the goal of systematization, to improve the manageability of the task in the system.

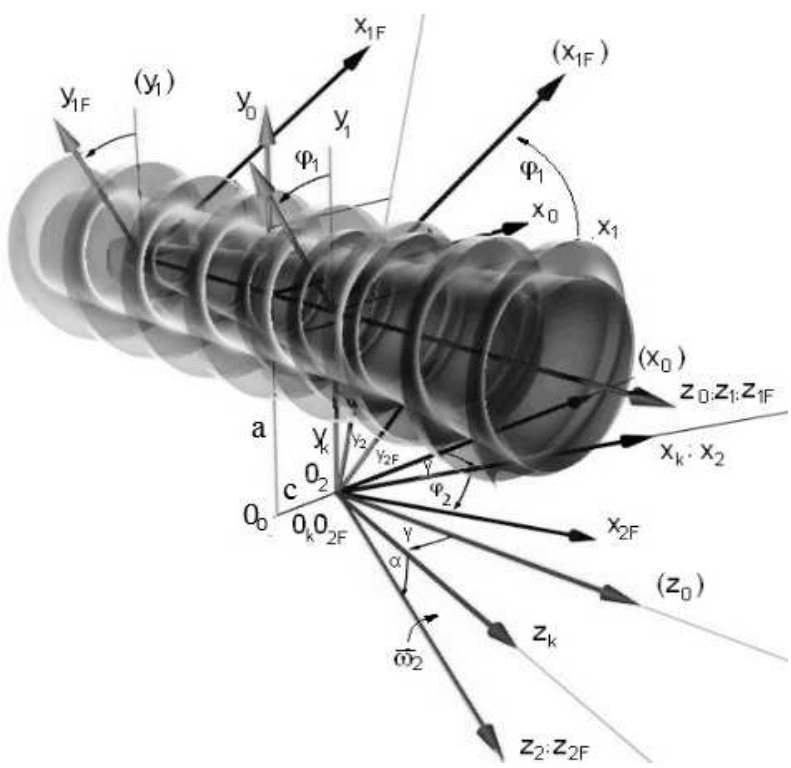

Fig. 7 The mathematical-kinematical model for the theory of manufacturing of the cylindrical and conical worms and their tools for uniaxial conical and cylindrical rolling surfaces [11]

\section{Result}

Worms from worm gearboxes for high power transmition have been machined by grinding wheel in a modern machining plants. From the rolling surfaces of the helicoidal elements of worm gear drive pairs according to their projective classification, the members of a class in the projective space have the same behavioral characterictics, so the were placed in a central collinear relationship, which futher developed the mathematical- kinematical model for unification. All this has been already described in the Euclidean space model, the geometrically correct machining leads to increased accuracy informing the operating surface of the conical worms.

In our further developed mathematical-kinematical geometric model of manufacturing process in case of changing distance of axes $\left(a_{0} \pm p \cdot \varphi_{1}\right)$, the elliptical errors have been eliminated. The geometrically correct manufacturing of conical worm surfaces, so elements of spiroid drives has become possible, as it can be seen in Figure 8 . 
The above model's geometrical development requires the construction of a new $\mathrm{NC}$ machine, which is also suitable of transverse movement with the grinding wheel in case of manufacturing.

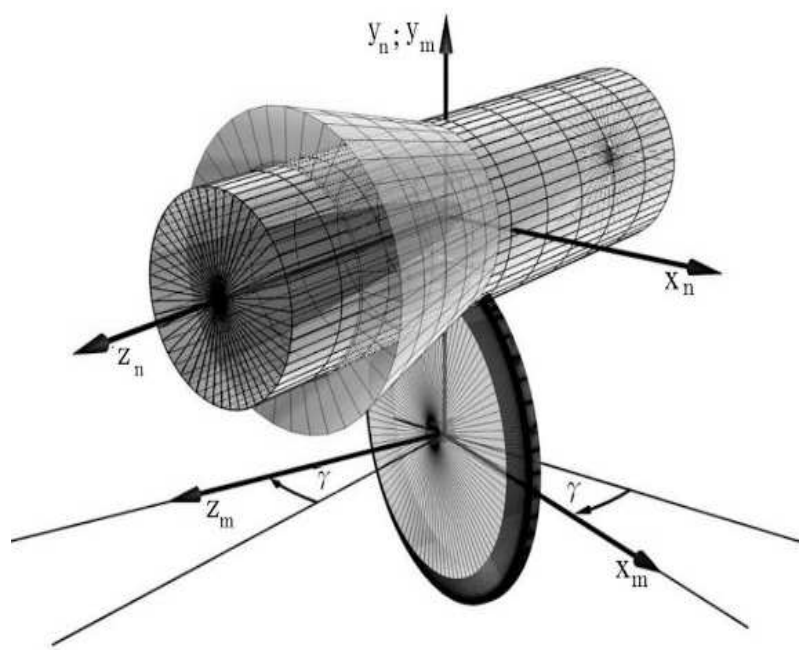

Fig. 8 The locating of the rolling surfaces of the cylindrical and conical worms mounted the same shaft in case of manufacturing with grinding wheel [11]

\section{Summary}

Based on the knowledge of projective geometry using the Dudás's mathematical-kinemathical model, the further developed using the projective geometrical model has been demonstrated. The necessy examination has been outlined about axoides of conical and cylindrical worms gear drives. The central collinear relationship has been determined between the rolling cone and the rolling cylinder in case of a rotating common axis. The examination has been performed to confirm that the matrix representation of the central collinearly relationship between the rolling cone and rolling cylinder surfaces is suitable to fit ProMat model. The improved mathematical-kinematical model ensures geometrically accurate production of conical worm.

\section{References}

[1] BALAJTI, ZS., ÁBEL, J. (2014) Applying projective geometry in design of worm manufacturing, Key Engineering Materials. Vol. 581. pp. 77-81. ISSN 1662-9795. DOI 10.4028/www.scientific.net/KEM.581.777. Trans Tech Publication, Switzerland.

[2] BUCUR, B., MÁTÉ, M. (2011) Theoretical Peculiarities Regarding the Definition and Representation of the Rolling Surfaces by a Bevel Worm Gear with the Crown Gear of Inverted Conicity, The 5th Edition of the Interdisciplinarity in Engineering International Conference in "Petru Maior” University. pp.165-169. http://inter- eng.upm.ro/2011/proceedings.html Indexed in Ulrich's Periodicals Directory ${ }^{\mathrm{TM}}$ (U.S.). German National Library of Science and Technology (TIB), Tîrgu Mureş. Romania.

[3] DRAHOS, I. (1972). The Motion Geometry Bases of Tool Geometry, Technical Study Book in Hungarian, p. 100. Institute of Continuing Education of the Technical University of Budapest at the Technical Textbook Publisher Company, Budapest.

[4] DUDÁS, I. (2004). The Theory \& Practice of Worm Gear Drives, Technical Professional Book, p. 320. ISBN 19039 96619. Penton Press Kogan Page, London.

[5] DUDÁS, I. (2016). The extension of the general mathematical model developed for helicodial surfaces to the whole system of manufacturing technology and production geometry (ProMAT), The International Journal of Advanced Manufacturing Technology. Vol. 86. Issue 5-8. pp. 15571572. Springer Print. ISSN 0268-3768, London.

[6] FORGÓ, Z., KAKUCS, A., MÁTÉ, M., TOLVALY-ROSCA, F. (2017) Development of Helical Teethed Involute Gear Meshed with a Multi-Edge Cutting Tool Using a Mixed Gear Teeth Modelling Method, Procedia Engineering Journal, Vol. 5. No. 2. pp. 1-6. Elsevier. ISSN 1877-7058, Switzerland.

[7] KRAL, J., KRAL, J. (2013) Calculation of the shape of tool cutting edge for production of involute worm Advances, Science and Technology Research Journal. Vol. 7. No. 17. pp. 1-5. DOI: 10.5604/20804075.1036988, Slovakia.

[8] LITVIN., F. L., FUENTES., A. (2004) Gear Geometry and Applied Theory, Technical Professional Book. pp. 44-58. Technical Cambridge University Press. ISBN 052181517 7, United Kingdom.

[9] MÁTÉ, M., HOLLANDA, D., TOLVALYROSCA, F., POPA-MÜLLER, I. (2013) The localization of the contact patch by cylindrical gear having an Archimedean tooth line using the method of setting the tangential displacement, OGÉT. Conference Proceedings. pp. 265-268. ISSN 2068-1267, Arad.

[10] ÓVÁRINÉ BALAJTI., ZS. (2007) Development of production geometry of kinematical drive pairs, $P h D$ dissertation. p. 126. (first drafting in Hungarian), Miskolc.

[11] ÓVÁRINÉ BALAJTI., ZS. (2016) Production Geometric Development of Meshing Surface Pairs, With Descriptive Geometric Application, 
Habilitation Thesis Book. p. 78. (first drafting in Hungarian), Miskolc.

[12] PETRICH G. (1979) Descriptive geometry, Technical Professional Book. p. 413. ISBN 96317 3814 0, Budapest.

[13] RACHKOVSKAYA., G. S., KHARABAYEV., Y. N. (2009) Geometric modelling and computer graphics of kinematic ruled surfaces on the base of complex moving one axoid along another (one-sheet hyperboloid of revolution as fixed and moving axoids,
WSCG 2009. 17-th International Conference on Computer Graphics. Visualization and Computer Vision. pp. 31-34. ISBN978-80-86943-95-4, Pilsen. Czech Republic.

[14] KUNDRAK, J., FELHÖ, CS. (2016) 3D Roughness Parameters of Surfaces Face Milled by Special Tools, Manufacturing Technology Journal for Science, Researche and Production. Vol. 16, No. 3. pp. 19-20. ISSN 1213-2489, Czech Republic. 